\title{
Thermal and mechanical analysis of the Wendelstein 7-X cryo-vacuum pump plug-in
}

\author{
Z. Wang ${ }^{\mathrm{a}}$, G. Ehrke ${ }^{\mathrm{b}}$, B. Mendelevitch ${ }^{\mathrm{a}}$, J. Boscary ${ }^{\mathrm{a}}$, R. Stadler ${ }^{\mathrm{a}}$, W7-X Team ${ }^{\mathrm{b}}$ \\ ${ }^{a}$ Max-Planck-Institut für Plasmaphysik, Bolzmannstr. 2, 85748 Garching, Germany \\ ${ }^{b}$ Max-Planck-Institut für Plasmaphysik, Wendelsteinstr. 1, 17491 Greifswald, Germany
}

\begin{abstract}
The cryo-vacuum pump (CVP) system controls the plasma density by condensing undesirable gases together with a set of turbo molecular pumps. One CVP will be installed under each of the 10 units of the actively cooled divertor in Wendelstein7-X for the long pulse operation up to $30 \mathrm{~min}$. duration. Each CVP is operated with supercritical Helium (ScHe) at $4 \mathrm{~K}$ and liquid nitrogen (LN2) at $80 \mathrm{~K}$ fed by a plug-in, which is installed inside a $\mathrm{W7-X}$ port of the plasma vessel. The plug-in made of stainless steel provides for the vacuum boundary between the plasma chamber and the torus hall atmosphere. 4 transfer lines $(12 \times 1 \mathrm{~mm})$ are positioned inside the plug-in: 2 for the inlet/outlet of ScHe and 2 for the inlet/outlet of LN2, respectively. The design has to guarantee the feeding at the specified temperature of ScHe and LN2 while minimizing thermal losses and thermal interactions between pipes. Inside the plug-in the vacuum level is $10^{-3} \mathrm{~Pa}$ at $\mathrm{RT}$ and $10^{-5} \mathrm{~Pa}$ during operation. The pipes of the ScHe are shielded with a multi-layer super-insulation (MLI). The cryogenic feed lines are protected with a cryo-shield against thermal loads in the port as well as in the plasma vessel. The thermal analysis confirmed the efficiency of the thermal shielding design, which keeps temperatures of ScHe and LN2 pipes within operation limits. During baking, the plasma vessel is heated to $150{ }^{\circ} \mathrm{C}$ while the cryostat remains close to $\mathrm{RT}$. The port and transfer lines are equipped with bellows to accommodate this situation. But the plugin is rigid and the resulting bend induces mechanical loads on the flanges between port and plugin. The mechanical analysis confirmed that the selected bolts and pretension conditions guarantee the tightness of the flange during baking.
\end{abstract}

Index Terms-Stellarator, Wendelstein 7-X, cryopump, thermal analysis, mechanical analysis

\section{INTRODUCTION}

$\mathrm{T}$ he stellarator Wendelstein7-X (W7-X) is a superconducting fusion device with five-fold magnetic field symmetry. Each of the 5 similar machine modules is made of two-flip symmetric half modules [1]. For the second operation phase (OP2) with high power and pulse duration up to $30 \mathrm{~min}$. an actively-cooled divertor system with a cryo vacuum pump (CVP) system will be installed. The divertor is made of ten discrete divertor units installed, above and below the helical axis of the plasma [2]. In total there are 10 identical

Z. Wang is with the Max Planck Institute for Plasma Physics, Garching, Germany (e-mail: zhw@ipp.mpg.de).

B. Mendelevitch, J. Boscary, R. Stadler, are with the Max Planck Institute for Plasma Physics, Garching, Germany.

G. Ehrke is with the Max Planck Institute for Plasma Physics, Greifswald, Germany.
CVPs, one CVP for each of the 10 divertor units, which is installed in the divertor volume below the horizontal target modules (Fig.1).

A CVP is operated with supercritical Helium $(\mathrm{ScHe})$ at $4 \mathrm{~K}$ and liquid nitrogen (LN2) at $80 \mathrm{~K}$ fed by a plug-in, which is installed inside a W7-X port of the plasma vessel (PV). The plug-in made of stainless steel provides for the vacuum boundary between the PV and the torus hall atmosphere. The outer dimensions of the plug-in are: $\sim 2 \mathrm{~m}$ long and $\sim \varnothing 0 \mathrm{~mm}$. 4 transfer lines are positioned inside the plug-in: 2 for the inlet/outlet of $\mathrm{ScHe}$ and 2 for the inlet/outlet of $\mathrm{LN} 2$, respectively (Fig.2). The dimension of the pipes is $12 \times 1 \mathrm{~mm}$. On the supply interface side, the pipes are equipped with bellows to compensate the thermal elongation during operation. On the PV side, the connection to the CVP is equipped with flexible hoses to allow compensating of assembly tolerances and to accommodate the displacement of the PV during operation. The design needs to guarantee the feeding at the specified temperature of ScHe and LN2 while minimizing thermal losses and thermal interactions between pipes. Inside the plug-in the vacuum level is $10^{-3} \mathrm{~Pa}$ at room temperature (RT) and $10^{-5} \mathrm{~Pa}$ during operation. During baking, the relative displacement due to the difference in thermal expansion between the port and the cryostat generates mechanical load that could damage the plug-in and endanger the CVP feeding. This paper presents the thermal and mechanical analysis performed with ANSYS [3] to check the selected design of the plug-in of the CVP.

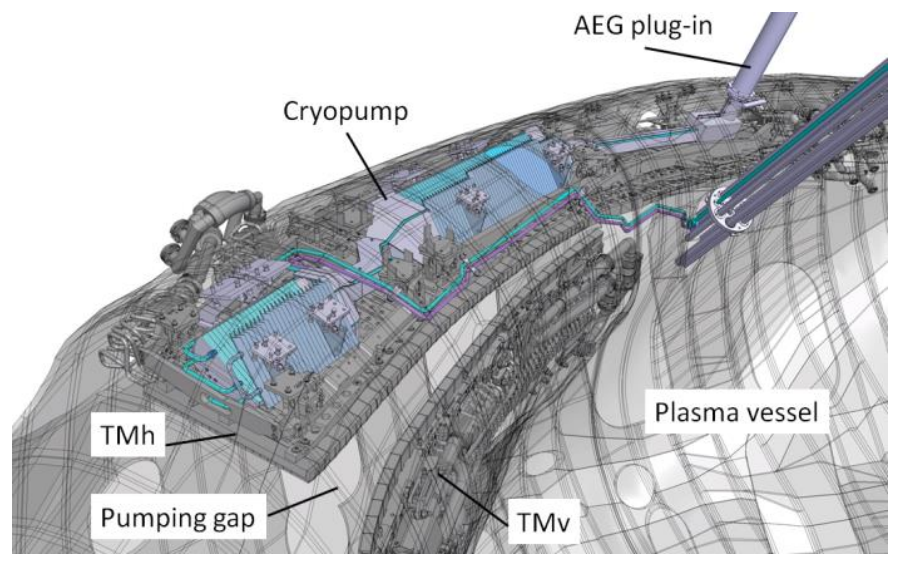

Fig. 1. CAD view of the CVP of one divertor unit (TMh: horizontal target module, TMv: vertical target module) and plug-in for the CVP. 


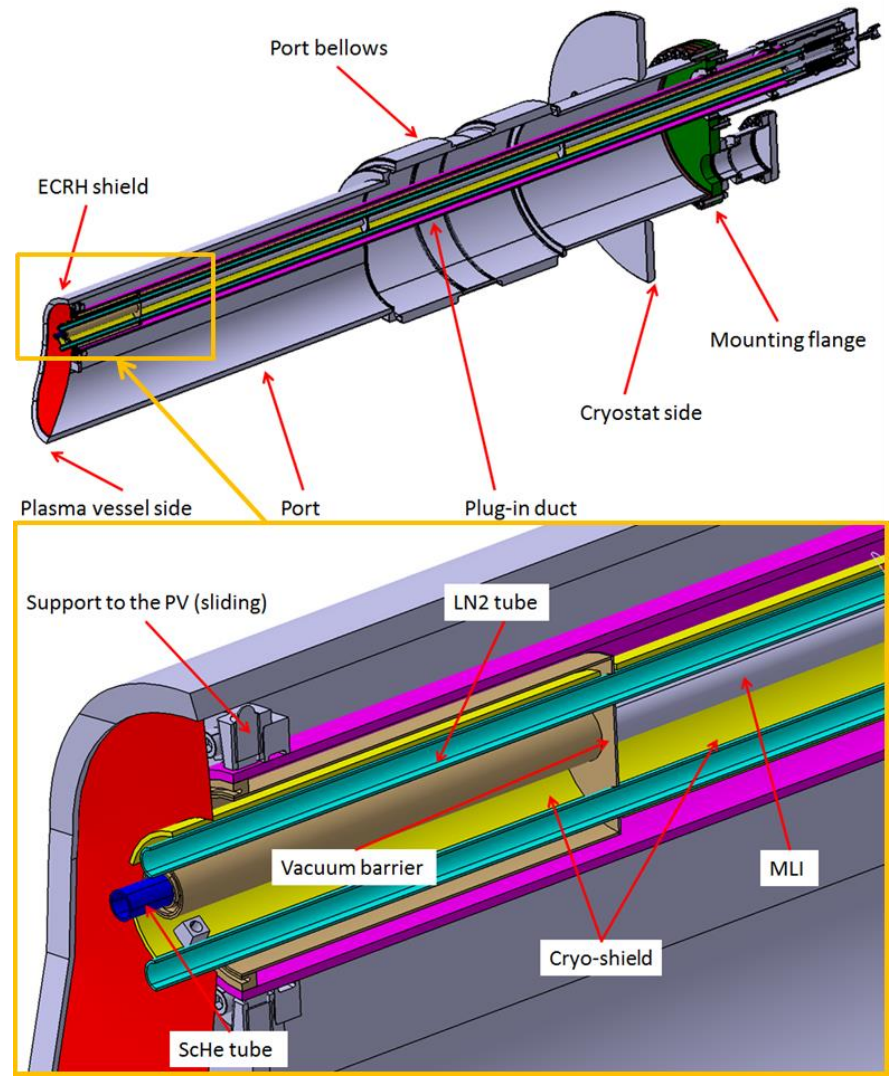

Fig. 2. View of the port and plug-in with transfer lines.

\section{THERMAL ANALYSIS}

The thermal design of the plug-in has to minimize heat load to ScHe and LN2, in order to guarantee the CVP operation, taking into account the following boundary conditions:

1. The plug-in is supported by a flange placed outside the cryostat, which has a direct conduction path to the RT environment; the extension of the flange also generates heat radiation to the plug-in.

2. During operation, the port connecting the PV and the cryostat is heated by the PV, which could reach a maximal temperature of $80^{\circ} \mathrm{C}$ and radiates to the plug-in.

3. The main heating system of $\mathrm{W} 7-\mathrm{X}$ is the Electron Cyclotron Resonance Heating (ECRH). To prevent ECRH heat load to the plug-in, a shield is positioned at the opening of the port at the PV side; this shield is heated and generates heat radiation.

Following design features have been employed to reduce the effects of heat conduction and radiation:

1. A multi-layer super insulation (MLI) is applied on the ScHe pipes to reduce the radiation heat load.

2. A cryo-shield welded to the LN2 pipe protects the ScHe pipes with the cost of increasing LN2 heat load; therefore on the outer surface of the cryo-shield, another MLI is used to reduce the radiation to the LN2 system.

3. The plug-in separates the PV vacuum from the cryo-distribution one; the barrier plate has a U-neck section to lengthen the conduction path and to shield against some heat radiation.

4. The support between the cryo-shield and the plug-in duct has only line-contacts with both sides, to reduce the heat conduction; in this analysis the support is ignored.

Due to the low pressure $\left(10^{-3} \mathrm{~Pa}\right.$ at $\mathrm{RT}$ and $10^{-5} \mathrm{~Pa}$ during operation), the convection of the remaining gas is ignored.

The inner surface of the pipes is set to the constant temperatures of $4 \mathrm{~K}$ and $80 \mathrm{~K}$ for the $\mathrm{ScHe}$ and $\mathrm{LN} 2$ pipes, respectively. The shield at the opening to the $\mathrm{PV}$ heated by the ECRH is set at $137^{\circ} \mathrm{C}$, the port inside the cryostat is set at $80^{\circ} \mathrm{C}$, and the part outside the cryostat is at RT, the temperature gradient between these two parts is ignored. This approach is very conservative. All stainless steel surfaces have an emissivity of 0.3 , and the MLI made of aluminum foils has 0.03 . Both conduction and radiation heat transfer are included in the analysis.

Radiation setup is critical for this analysis because the ECRH shield and the port transmit their heat by radiation, meanwhile the cryo-shield and MLI reduce mainly the radiation heat load. A radiation network is shown in Fig. 3, the radiation heat from the ECRH shield and the port has to pass the plug-in duct and the cryo-shield to reach the coolant. On the PV side, the environment is assumed to be the $\mathrm{PV}$; and on the cryo-distribution side, the environment is at RT.

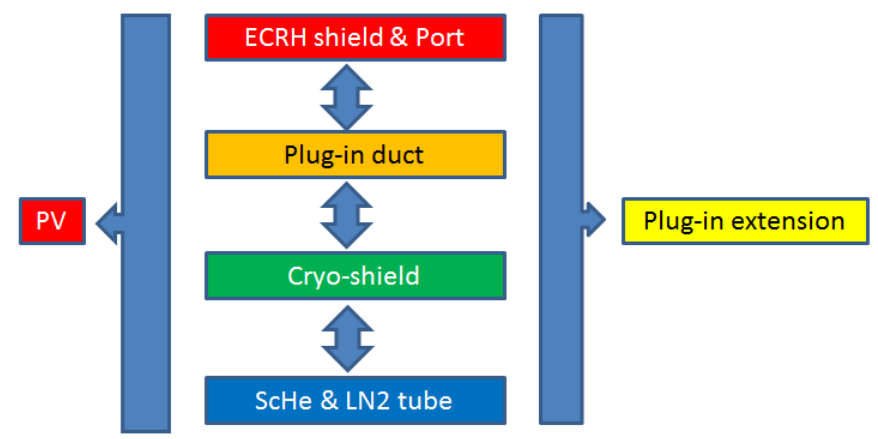

Fig. 3. Scheme of heat radiation modeling in plug-in.

The calculation of the global temperature distribution is shown in Fig. 4. Outcome of the calculations can be summarized as follows:

1. The plug-in duct has the similar temperature distribution as the port itself. This result confirms the need for the installation of a radiation shield to prevent the direct conduction path between the plug-in duct and the cryogenic components.

2. The installation of the MLI on the outer surface allows a quite uniform temperature of the cryo-shield similar to the LN2 tubes; all components placed inside the cryo-shield are efficiently protected against the hot plug-in duct (Fig.5).

3. The temperature gradient at the $\mathrm{ScHe}$ tube connector is due to the conducted heat to the required vacuum barrier despite the shielding by the LN2 pipes and the extension of the conduction path with the U-neck design (Fig.6).

4. The environment radiation affects only the components placed close to the opening of the cryo-shield. 
5. The heat load to the $\mathrm{ScHe}$ system is $4 \mathrm{~W}$, and $13 \mathrm{~W}$ to the LN2. Compared to the CVP heat loads [4], these values can be accepted.

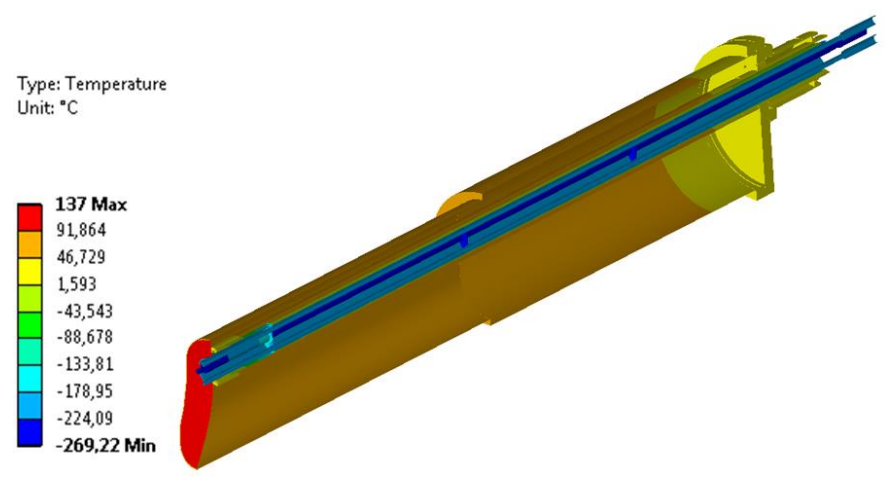

Fig. 4. Global temperature distribution of the plug-in and port

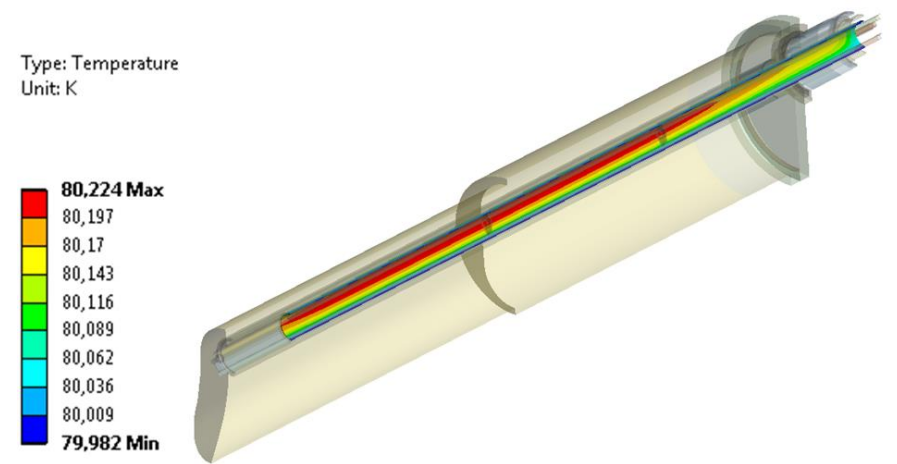

Fig. 5. Temperature distribution of the cryo-shield

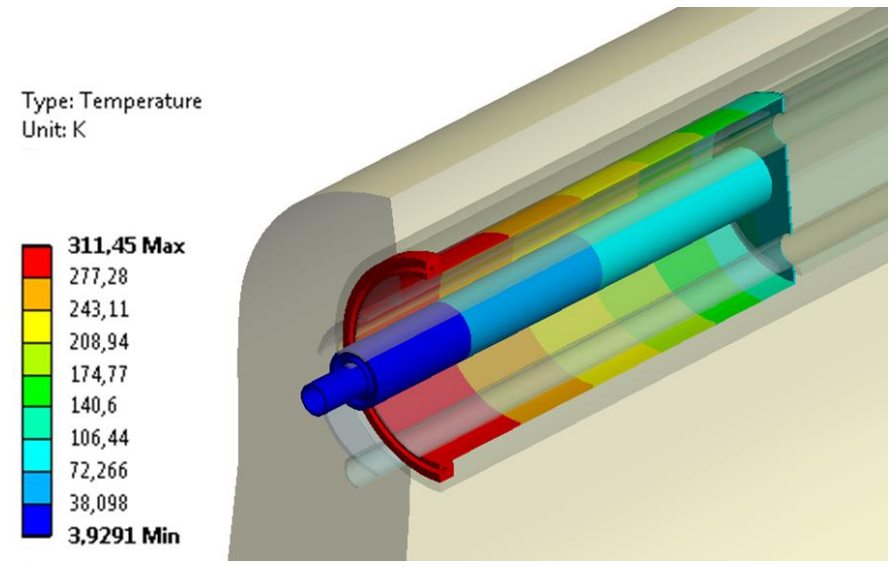

Fig. 6. Temperature distribution of the vacuum barrier

\section{MECHANICAL ANALYSIS}

In the baking scenario, the PV is heated to $150^{\circ} \mathrm{C}$ while the cryostat remains close to RT. The expansion of the PV produces a relative displacement between the PV and the cryostat. The corresponding mechanical loads have been simulated in the W7-X global model of the cryostat system (GMCS) [5]. The port is equipped with bellows to accommodate this situation. The plug-in is rigid and will bend due to the pushing force from the PV, this movement will mechanically load the bolt connection of the plug-in. The mechanical analysis aims to check the impact.

Due to the installation of flexible connections on both sides, the ScHe and LN2 pipes do not have bending problems. Therefore the mechanical model contains only the flange to the cryostat, the plug-in duct and the bolt/screw connected port \& plug-in flanges (Fig.7). Many non-linear contacts are defined to simulate real conditions as much as possible. For example, the frictional contact between the flanges allows a possible detachment, which results in the mechanical loads of the bolts.

In the GMCS, the plug-in is not included. The mechanical loads needs to be deduced from the results of the PV, port and cryostat. In Fig. 8, a local Cartesian coordinate system is defined to describe the displacement load. On the cryostat side, the plug-in is fixed while the sliding support on the PV side pushes the plug-in in the $\mathrm{Y}$ and $\mathrm{Z}$ directions. There are 10 plug-ins distributed in different positions and oriented in different directions around the machine. The name of the port is AEG, and are numbered from $X 0$ and $X 1$, where $X=1$ to 5 for the five machine module (AEG10 51) A parametric study has been performed to find the worst case (see Table. I). According to this table, AEG11 has the largest Y-displacement, both Y and $\mathrm{Z}$ displacements are large for AEG21, and AEG51 has similar values.

TABLE I

MECHANICAL LOAD OF CVP PLUG-IN

\begin{tabular}{lcc}
\hline \hline Number & Y-displacement $(\mathrm{mm})$ & Z-displacement $(\mathrm{mm})$ \\
\hline AEG10 & 14.7 & 2.8 \\
AEG11 & 18.0 & 2.8 \\
AEG20 & 15.2 & 2.8 \\
AEG21 & 17.6 & 5.6 \\
AEG30 & 14.7 & 0.7 \\
AEG31 & 15.7 & 4.8 \\
AEG40 & 15.5 & 3.2 \\
AEG41 & 15.8 & 5.5 \\
AEG50 & 15.1 & 3.5 \\
AEG51 & 16.9 & 5.6 \\
\hline \hline
\end{tabular}

The pretension of the bolt and screw is another important issue. Obviously with higher value, the plug-in can hold its position better against the $\mathrm{PV}$, and improve the fatigue performance, but the bolts will take higher loads; if the pretension is loosed, then the flanges may open and the vacuum boundary is broken. Therefore, the pretension is also a parameter to study, different percentages of the yield strength have been chosen to represent the pretension, as Table. II shows.

TABLE II

PRETENSION OF BOLT/SCREW

\begin{tabular}{ccc}
\hline \hline $\begin{array}{c}\text { Ratio (pretension } \\
\text { stress/yield strength) }\end{array}$ & Bolt load (N) & Screw load (N) \\
\hline $2.5 \%$ & 334 & 214 \\
$5 \%$ & 668 & 428 \\
$10 \%$ & 1335 & 855 \\
$20 \%$ & 2671 & 1709 \\
$40 \%$ & 5341 & 3418 \\
$60 \%$ & 8012 & 5127 \\
$80 \%$ & 10682 & 6836 \\
\hline \hline
\end{tabular}


At first, the pretension is set at $40 \%$ of the yield strength, the displacement loads are applied for all 10 plug-ins as in Table I. Table. III gives the bolt/screw connection results for each plug-in. The plug-in is bent; the moment tightens the bolt/screw on one side and compresses them on the other side. Due to the pretension, the bolt/screw is always under tension, but some of them have higher loads.

TABLE III BOLT/SCREW CONNECTION RESULTS

\begin{tabular}{ccc}
\hline \hline Number & Peak bolt load (N) & Peak screw load (N) \\
\hline AEG10 & 5705 & 3856 \\
AEG11 & 5981 & 4009 \\
AEG20 & 5734 & 3876 \\
AEG21 & 6093 & 4037 \\
AEG30 & 5632 & 3820 \\
AEG31 & 5875 & 3937 \\
AEG40 & 5781 & 3896 \\
AEG41 & 5923 & 3956 \\
AEG50 & 5765 & 3886 \\
AEG51 & 6022 & 4005 \\
\hline \hline
\end{tabular}

As shown in Table III, the most critical plugin is AEG21 and is considered for next analysis, the resulting working loads for various pretension forces are summarized in Fig.9. When the pretension is lower than $5 \%$ of the yield strength, the minimum working load of the bolt/screw is 0 , which means that the connection is lost. The pretension has to be higher than $10 \%$ of the yield strength.

There is a seal ring made of $\mathrm{Cu}$ between the two flanges which is compressed by $0.1 \mathrm{~mm}$. If the gap between the two flanges is smaller than $0.1 \mathrm{~mm}$, the flange is considered to be tight. Fig.10 shows the gap distributions for different pretension forces. The pretension higher than $10 \%$ of the yield strength keeps the vacuum tightness.

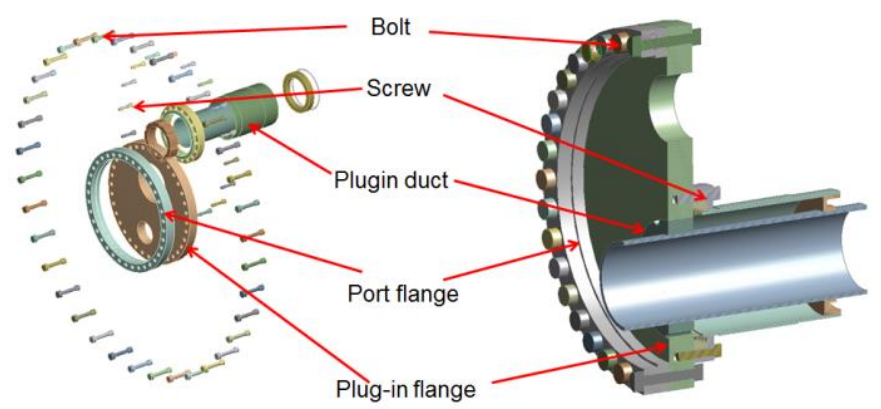

Fig. 7. Mechanical plug-in model

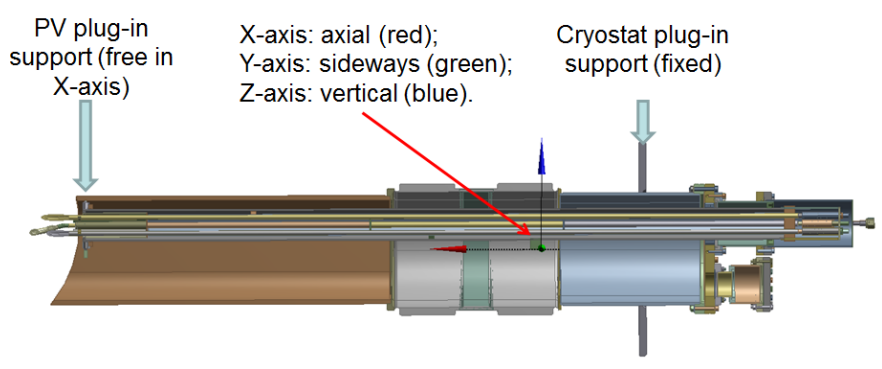

Fig. 8. Mechanical plug-in model and boundary conditions

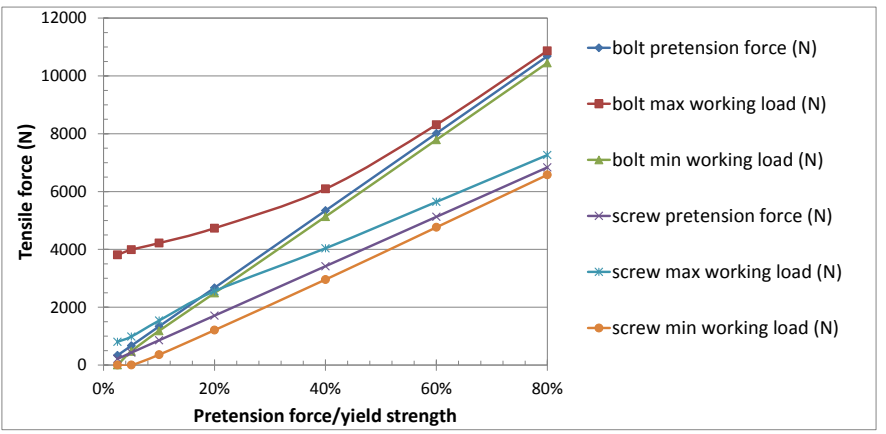

Fig. 9. Evolution of the bolt/screw pretension and working load.
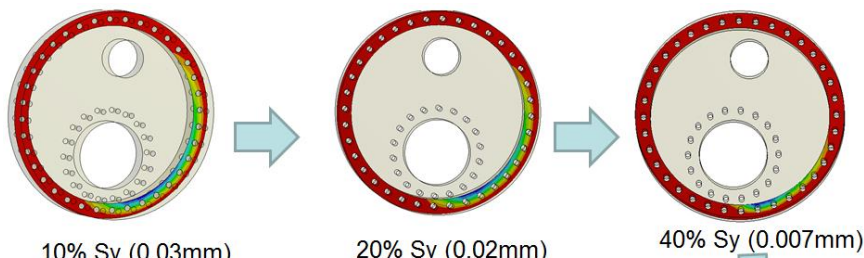

$20 \%$ Sy $(0.02 \mathrm{~mm})$

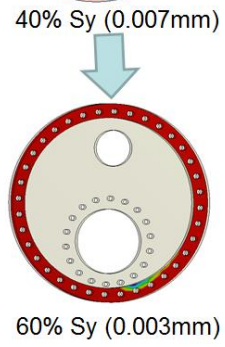

Fig. 10. Gap between the flanges with different pretension forces

\section{CONCLUSIONS}

The transfer lines of SCHe and LN2, which operate at $4 \mathrm{~K}$ and $80 \mathrm{~K}$, respectively, are connected to the CVP of W7-X in each of the 10 divertor units by a plug-in. The plug-in design uses cryo-shields and MLI to protect the ScHe and LN2 cooling pipes against heat loads. The thermal analysis performed with ANSYS confirmed the efficiency of the thermal shielding design, which keeps temperatures of ScHe and LN2 pipes within operation limits. Heat loads to the ScHe system of $4 \mathrm{~W}$ and $13 \mathrm{~W}$ to the LN2 are acceptable. The mechanical analysis has been performed for the worst case of baking operation. The difference between the plasma vessel heated to $150{ }^{\circ} \mathrm{C}$ and the cryostat close to RT produces the relative displacement between the PV and the cryostat. The port and transfer lines are equipped with bellows to accommodate this situation. But the plugin is rigid and the resulting bend induces mechanical loads on the flanges between port and plugin. The mechanical analysis confirmed that the selected bolts and pretension 
conditions guarantee the tightness of the flange during baking.

\section{REFERENCES}

[1] H. Renner, et al., "The capabilities of steady-state operation at the stellarator W7-X with emphasis on divertor design", Nucl. Fusion 40 (6) (2000) 1083-1093.

[2] J. Boscary, A. Peacock, R. Stadler, B. Mendelevitch, H. Tittes, et al., "Actively water-cooled plasma facing components of the Wendelstein 7-X stellarator”, Fus. Sci. Tech. 64 (2013) 263-268.

[3] ANSYS, 17.0, 2015,

[4] J. Zhu, et al., "Refined multiphysics analysis of W7-X cryopumps", presented at SOFE2017.

[5] Paul van Eeten, et al., "Features and analyses of W7-X cryostat system FE model”, Fus. Eng. and Des., vol. 96-97, Oct. 2015, pp. 369-372. 